\title{
396.
}

\section{ON A CERTAIN ENVELOPE DEPENDING ON A TRIANGLE INSCRIBED IN A CIRCLE.}

[From the Quarterly Journal of Pure and Applied Mathematics, vol. Ix. (1868), pp. $31-41$ and $175-176$.

Considering a triangle and the circumscribed circle, and from any point of the circle drawing perpendiculars to the sides of the triangle; the feet of the three perpendiculars lie on a line; and (regarding the point as a variable point on the circle) the envelope of the line is a curve of the third class, having the line infinity for a double tangent, and being therefore a curve of the fourth order with three cusps, see Steiner's paper "Ueber eine besondere Curve dritter Klasse und vierten Grades," Crelle, t. LIII. (18.57), pp. 231-237, which contains a series of very beautiful geometrical properties.

$\mathrm{Mr}$ Greer, in a paper in the last volume of the Journal, has expressed the equation of the line in a very elegant form, viz. if $\alpha, \beta, \gamma$ are the perpendicular distances of the point from the sides of the triangle; $A, B, C$ the angles of the triangle; $(\lambda, \mu, \nu)=\left(\frac{\cos A}{\alpha}, \frac{\cos B}{\beta}, \frac{\cos C}{\gamma}\right) ;$ and $(X, Y, Z)$ certain current coordinates, viz. these are the perpendicular distances from the sides, multiplied by $\sin A \tan A$, $\sin B \tan B, \sin C \tan C$ respectively; then the equation of the line is

$$
X \lambda(\lambda-\mu)(\lambda-\nu)+Y \mu(\mu-\nu)(\mu-\lambda)+Z \nu(\nu-\lambda)(\nu-\mu)=0
$$

where the parameters $\lambda, \mu, \nu$ are connected by the equation $\lambda \tan A+\mu \tan B+\nu \tan C=0$, or say by the equation

$$
\lambda \mathrm{a}+\mu \mathrm{b}+\nu \mathrm{c}=0
$$


We have a cubic equation in $(\lambda, \mu, \nu)$ with coefficients which are linear functions of $(X, Y, Z)$, and the required equation is that obtained by equating to zero the reciprocant of this cubic function, the facients of the reciprocant being the $(a, b, c)$ of the linear relation; the reciprocant is of the degree 6 in $(a, b, c)$ and of the degree 4 in the coefficients of the cubic function, that is in $(X, Y, Z)$. But I remark that the equation in $(\lambda, \mu, \nu)$, regarding these quantities as coordinates, is that of a cubic curve having a node at the point $\lambda=\mu=\nu$, or say the point $(1,1,1)$; the corresponding value of $\lambda \mathrm{a}+\mu \mathrm{b}+\nu \mathrm{c}$ is $=\mathrm{a}+\mathrm{b}+\mathrm{c}$, and the reciprocant consequently contains the factor $(a+b+c)^{2}$, or dividing this out, the equation is only of the degree 4 in $(a, b, c)$. The equation of the curve thus is

$$
\frac{1}{(\mathrm{a}+\mathrm{b}+\mathrm{c})^{2}} \text { recip. }\{X \lambda(\lambda-\mu)(\lambda-\nu)+Y \mu(\mu-\nu)(\mu-\lambda)+Z \nu(\nu-\lambda)(\nu-\mu)\}=0,
$$

being of the degree 4 in (a, b, c), and also of the degree 4 in $(X, Y, Z)$, that is, treating $(X, Y, Z)$ as current coordinates, the envelope is as above stated a curve of the fourth order.

A symmetrical method for finding the reciprocant of a cubic function was given by Hesse, see my paper "On Homogeneous Functions of the Third Order with Three Variables," Camb. and Dubl. Math. Jour., vol. I. (1846), pp. 97-104, [35]; the developed expression there given for the reciprocant is however erroneous; the correct value is given in my "Third Memoir on Quantics," Phil. Trans., vol. cxlvi. (1856), see the Table 67, p. $644,[144]$ and we have only in the table to substitute for $(\xi, \eta, \zeta)$ the quantities (a, b, c), and for $(a, b, c, f, g, h, i, j, k, l)$ the coefficients of the cubic function of $(\lambda, \mu, \nu)$, viz. multiplying by 6 in order to avoid fractions, these are

$$
\begin{aligned}
& (a, b, c, \quad f, \quad g, \quad h, \quad i, \quad j, k, \quad l \quad) \\
& =(6 X, 6 Y, 6 Z,-2 Y,-2 Z,-2 X,-2 Z,-2 X,-2 Y, X+Y+Z)
\end{aligned}
$$

respectively. The substitution might be performed as follows, viz. for the coefficient of $\mathrm{a}^{6}$, we have

$$
\left.\begin{array}{l}
b^{2} c^{2}+1.1296 Y^{2} Z^{2}+1296 \\
b c f i-6.144 Y^{2} Z^{2}-864 \\
b i^{3}+4 .-48 Y^{3}-192 \\
c f^{3}+4 .-48 Y^{3} Z-192 \\
f^{2} i^{2}-3 . \quad 16 Y^{2} Z^{2}-48
\end{array}\right\}=-192 Y Z(Y-Z)^{2}
$$

and so for the other coefficients; but I have not gone through the labour of performing the calculation. Omitting the numerical factor -192 , the coefficients of $a^{6}, b^{6}, c^{6}$ are of course

$$
Y Z(Y-Z)^{2}, Z X(Z-X)^{2}, X Y(X-Y)^{2}
$$

C. VI. 
and I find also that the coefficient of $\mathrm{b}^{5} \mathrm{c}$ (the factor -192 being omitted) is

$$
=Z X\left(3 X^{2}+3 Z^{2}+3 Y Z-6 Z X+5 X Y\right) \text {, }
$$

whence those of the terms $c^{5} \mathrm{a}, \& c$. are also known.

I denote the result as follows:

$$
\left(Y Z(Y-Z)^{2}, Z X(Z-X)^{2}, X Y(X-Y)^{2}, \ldots \chi(\mathrm{a}, \mathrm{b}, \mathrm{c})^{6}=0 ;\right.
$$

this equation divides as we have seen by $(a+b+c)^{2}$, and the quotient is

$$
\left(Y Z(Y-Z)^{2}, Z X(Z-X)^{2}, X Y(X-Y)^{2}, \ldots(a, b, c)^{4}=0 ;\right.
$$

and it may be remarked that the coefficient of $b^{3} c$ in this quartic function of $(a, b, c)$ is

$$
=Z X\left(X^{2}+Z^{2}+3 Y Z-2 Z X+5 X Y .\right.
$$

The last mentioned equation, if the calculation were completed, would be analytically the best form for the equation of the envelope; but in view of what follows, I will change it by writing $\mathrm{a} x, \mathrm{~b} y, \mathrm{c} z$ in place of $(X, Y, Z) ; x$ is therefore $=\frac{1}{\tan A} X$, that is, it is = perpendicular distance $\times \sin A$; or, what is the same thing, the new coordinates $(x, y, z)$ are proportional to the perpendicular distances from the sides, each distance divided by the perpendicular distance of the side from the opposite angle, the equation of the line infinity is thus $x+y+z=0$. I write also $(a, b, c)=\left(\frac{1}{a}, \frac{1}{b}, \frac{1}{c}\right)$, that is, we have $(a, b, c)=(\cot A, \cot B, \cot C)$. The system of equations is therefore

$$
\begin{aligned}
& \frac{x}{a} \lambda(\lambda-\mu)(\lambda-\nu)+\frac{y}{b} \mu(\mu-\nu)(\mu-\lambda)+\frac{z}{c} \nu(\nu-\lambda)(\nu-\mu)=0, \\
& \frac{\lambda}{a}+\frac{\mu}{b}+\frac{\nu}{c}=0,
\end{aligned}
$$

giving for the envelope the equation

$$
b c y z(c y-b z)^{2}+c a z x(a z-c x)^{2}+a b x y(b x-a y)^{2}+\& c .=0 ;
$$

and in this function, corresponding to the term

$$
\mathrm{b}^{3} \mathrm{c} Z X\left(X^{2}+Z^{2}+3 Y Z-2 Z X+5 X Y\right) \text {, }
$$

we have the term

$$
a z x\left(b c^{2} x^{2}+a^{2} b z^{2}+3 a^{2} c y z-2 a b c z x+5 a c^{2} x y\right) .
$$

It may be noticed that, arranging in powers of $(x, y, z)$, the several portions of each coefficient are distinct literal functions; thus we see that the coefficient of $z^{3} x$ is $=a^{3} c+a^{3} b+$ other combinations of $(a, b, c)$ : this is material in order to the comparison of the foregoing equation of the envelope in a different form which will be presently mentioned. 
I proceed to find the tangential equation of the envelope. Representing the equation of the line by

we have

$$
\xi x+\eta y+\zeta z=0
$$

$$
\xi: \eta: \zeta=\frac{1}{a} \lambda(\lambda-\mu)(\lambda-\nu): \frac{1}{b} \mu(\mu-\nu)(\mu-\lambda): \frac{1}{c} \nu(\nu-\lambda)(\nu-\mu),
$$

or, what is the same thing,

$$
\xi: \eta: \zeta=\frac{1}{a} \quad \frac{\lambda}{\mu-\nu} \quad: \frac{1}{b} \quad \frac{\mu}{\nu-\lambda} \quad: \frac{1}{c} \frac{\nu}{\lambda-\mu},
$$

where as before

$$
\frac{\lambda}{a}+\frac{\mu}{b}+\frac{\nu}{c}=0
$$

and eliminating $\lambda, \mu, \nu$, we find

$$
a \xi(\eta-\zeta)^{2}+b \eta(\zeta-\xi)^{2}+c \zeta(\xi-\eta)^{2}=0 .
$$

In fact we find at once

$$
\begin{aligned}
a \xi(\eta-\zeta)^{2}: b \eta(\zeta-\xi)^{2}: c \zeta(\xi-\eta)^{2}= & (\mu-\nu) \lambda\left\{\frac{1}{b} \mu(\lambda-\mu)-\frac{1}{c} \nu(\nu-\lambda)\right\} \\
& :(\nu-\lambda) \mu\left\{\frac{1}{c} \nu(\mu-\nu)-\frac{1}{a} \lambda(\lambda-\mu)\right\} \\
& :(\lambda-\mu) \nu\left\{\frac{1}{a} \lambda(\nu-\lambda)-\frac{1}{b} \mu(\mu-\nu)\right\},
\end{aligned}
$$

and the sum of the three expressions on the right-hand side is

$$
=-(\mu-\nu)(\nu-\lambda)(\lambda-\mu)\left(\frac{\lambda}{a}+\frac{\mu}{b}+\frac{\nu}{c}\right)=0,
$$

which verifies the result just obtained.

The tangential equation of the envelope is thus

$$
a \xi(\eta-\zeta)^{2}+b \eta(\zeta-\xi)^{2}+c \zeta(\xi-\eta)^{2}=0,
$$

or the envelope is a curve of the third class having as a double tangent the line $\xi=\eta=\zeta$, that is the line infinity; in fact for these values the equation $\xi x+\eta y+\zeta z=0$ becomes $x+y+z=0$, which is the equation of the line infinity. The curve is therefore a curve of the fourth order, the equation of which is

$$
\frac{1}{(x+y+z)^{2}} \text { recip. }\left\{a \xi(\eta-\zeta)^{2}+b \eta(\zeta-\xi)^{2}+c \zeta(\xi-\eta)^{2}\right\}=0
$$


where the reciprocant in question may be calculated from the before mentioned table 67, viz. multiplying by 3 in order to avoid fractions, the coefficients of the table are

$$
\begin{aligned}
& (a, b, c, f, g, h, i, j, k, \quad l \\
= & (0,0,0, c, a, b, b, c, a,-a-b-c)
\end{aligned}
$$

respectively, and for the facients $(\xi, \eta, \zeta)$ of the table we have to write $(x, y, z)$. The expression of the reciprocant is

$$
=b^{2} c^{2} x^{6} \dashv c^{2} a^{2} y^{6}+a^{2} b^{2} z^{6}+\& c .
$$

and dividing by $(x+y+z)^{2}$ we have the equation of the envelope in the form

$$
b^{2} c^{2} x^{4}+c^{2} a^{2} y^{4}+a^{2} b^{2} z^{4}+\& c .=0,
$$

which must of course be identical with the former result

$$
b c y z(c y-b z)^{2}+c a z x(a z-c x)^{2}+a b x y(b x-a y)^{2}+\& c .=0 .
$$

Instead of discussing the curve of the third class

$$
a \xi(\eta-\zeta)^{2}+b \eta(\zeta-\xi)^{2}+c \zeta(\xi-\eta)^{2}=0,
$$

it will be convenient to write $(x, y, z)$ in place of $(\xi, \eta, \xi)$, and discuss the curve of the third order, or cubic curve

$$
U=a x(y-z)^{2}+b y(z-x)^{2}+c z(x-y)^{2}=0,
$$

which is of course a curve having a node at the point $(x=y=z)$, or say at the point $(1,1,1)$, and having therefore three inflexions lying in a line. The equation of the tangents at the node is found to be

$$
a(y-z)^{2}+b(z-x)^{2}+c(x-y)^{2}=0,
$$

that is, at the node the second derived functions of $U$ are proportional to

$$
(b+c, c+a, a+b,-a,-b,-c) \text {. }
$$

The equation of the Hessian may be found directly, or by means of the table, No. 61, in my memoir above referred to. It is as follows:

$$
\begin{aligned}
& (b+c)\{a(b+c)+2 b c\} x^{3} \\
+ & (c+a)\{b(c+a)+2 c a\} y^{3} \\
+ & (a+b)\{c(a+b)+2 a b\} z^{3} \\
- & \left(3 a^{2}+2 b c+2 c a+2 a b\right)\left(c y^{2} z+b y z^{2}\right) \\
- & \left(3 b^{2}+2 b c+2 c a+2 a b\right)\left(a z^{2} x+c z x^{2}\right) \\
- & \left(3 c^{2}+2 b c+2 c a+2 a b\right)\left(b x^{2} y+a x y^{2}\right) \\
+ & \left\{4\left(b c^{2}+b^{2} c+c a^{2}+c^{2} a+a b^{2}+a^{2} b\right)+6 a b c\right\} x y z=0 .
\end{aligned}
$$


I find that in the function $b^{2} c^{2} x^{6}+\& c$. the term in $z^{5} x$ is

$$
=a\left(4 a^{2} b+4 a^{2} c+4 a b^{2}+4 a b c-2 b^{2} c\right),
$$

whence in the function $b^{2} c^{2} x^{4}+\& c$. the term in $z^{3} x$ is

$$
=z^{3} x a\left(4 a^{2} b+4 a^{2} c+2 a b^{2}+4 a b c-2 b^{2} c\right),
$$

a portion whereof is $=4 z^{3} x\left(a^{3} b+a^{3} c\right)$; and we thus obtain the numerical factor $=4$, and thence the identity

$$
b^{2} c^{2} x^{4}+c^{2} a^{2} y^{4}+a^{2} b^{2} z^{4}+\& c .=4 b c y z(c y-b z)^{2}+\ldots+\& c .
$$

which equation I represent by

or

$$
A x^{3}+B y^{3}+C z^{3}+3\left(F y^{2} z+G z^{2} x+H x^{2} y+I y z^{2}+J z x^{2}+K x y^{2}\right)+6 L x y z=0,
$$

$$
(A, B, C, F, G, H, I, J, K, L \chi \chi x, y, z)^{3}=0,
$$

viz. writing for shortness

$$
M=b c+c a+a b,
$$

the values of the coefficients are as follows:

$$
\begin{array}{rcc}
A=3(b+c)(b c+M), & F=-c\left(3 a^{2}+M\right), & I=-b\left(3 a^{2}+M\right), \\
B=3(c+a)(c a+M), & G=-a\left(3 b^{2}+M\right), & J=-c\left(3 b^{2}+M\right), \\
C=3(a+b)(a b+M), & H=-b\left(3 c^{2}+M\right), & K=-a\left(3 c^{2}+M\right), \\
L=2(a+b+c) M-3 a b c . &
\end{array}
$$

I remark that the cubic having a node at the point $(1,1,1)$, the Hessian has at this point a node with the same tangents. The second derived functions for the Hessian are therefore at the node proportional to those of the cubic; it is easy to verify that we have in fact

$$
\begin{array}{ll}
A+H+J=(b+c) M, & L+F+I=-a M, \\
K+B+F=(c+a) M, & J+L+G=-b M, \\
G+I+C=(a+b) M, & H+K+L=-c M,
\end{array}
$$

these values give also

$$
\begin{aligned}
& A+K+G+2 L+2 J+2 H=0 \\
& H+B+I+2 F+2 L+2 K=0 \\
& J+F+C+2 I+2 G+2 L=0
\end{aligned}
$$

equations which merely express that the first derived functions vanish at the node. If, by these equations we express $A, B, C$ in terms of the other coefficients, and substitute these values in the equation of the Hessian, this may be expressed in the form

$$
\begin{aligned}
& (y-z)^{2}\{ \\
+ & L x+(2 F+I+L) y+(F+2 I+L) z\} \\
+ & (z-y)^{2}\{(G+2 J+L) x+\quad L y+(2 G+J+L) z\} \\
& \{(2 H+K+L) x+(H+2 K+L) y+\quad L z\}=0,
\end{aligned}
$$

a form which puts in evidence the node $(1,1,1)$. 
I write

$$
X=y-z, \quad Y=z-x, \quad Z=x-y,
$$

so that we have identically

$$
X+Y+Z=0
$$

and that the equation of the tangents at the node is

$$
a X^{2}+b Y^{2}+c Z^{2}=0
$$

I write also for shortness

$$
\begin{array}{ll}
F^{\prime}=2 F+I+L, & I^{\prime}=F+2 I+L, \\
G^{\prime}=2 G+J+L, & J^{\prime}=G+2 J+L, \\
H^{\prime}=2 H+K+L, & K^{\prime}=H+2 K+L
\end{array}
$$

the equation of the cubic is then

$$
U=a x X^{2}+b y Y^{2}+c z Z^{2},=0,
$$

and that of the Hessian is

$$
\tilde{H} U=X^{2}\left(L x+F^{\prime} y+I^{\prime} z\right)+Y^{2}\left(J^{\prime} x+L y+G^{\prime} z\right)+Z^{2}\left(H^{\prime} x+K^{\prime} y+L z\right) .
$$

Now observing that we have

we find

$$
\begin{aligned}
& L+3 a M=L-3(I+F+L)=-F^{\prime}-I^{\prime} \\
& L+3 b M=L-3(G+J+L)=-G^{\prime}-J^{\prime} \\
& L+3 c M=L-3(H+K+L)=-H^{\prime}-K^{\prime}
\end{aligned}
$$

$$
\begin{aligned}
\tilde{H} U+3 M U= & X^{2}\left(I^{\prime} Y-F^{\prime} Z\right) \\
& +Y^{2}\left(J^{\prime} Z-G^{\prime} X\right) \\
& +Z^{2}\left(K^{\prime} X-H^{\prime} Y\right)
\end{aligned}
$$

which shows that the function $\tilde{H} U+3 M U$ is a cubic function of $y-z, z-x, x-y$, decomposable therefore into three linear factors; and the equation $\tilde{H} U+3 M U=0$, is consequently that of the three lines drawn from the node to the three inflexions of the cubic (or the Hessian). We know also that the Hessian of the three lines is the pair of tangents at the node $\left.{ }^{1}\right)$, viz. that regarding any one of the variables $X, Y, Z$ as a linear function of the third of them (in virtue of the equation $X+Y+Z=0$ ), then that the cubic function of $X, Y, Z$ has $a X^{2}+b Y^{2}+c Z^{2}$ for its Hessian.

1 Taking as the canonical form of a nodal cubic $U=x^{3}+y^{3}+6 l x y z=0$, then we have $\tilde{H} U=x^{3}+y^{3}-2 l x y z=0$; $x^{3}+y^{3}=0$ is the equation of the lines from the node to the inflexions, and the Hessian of the binary cubic $x^{3}+y^{3}$ is $x y$, where $x y=0$ is the equation of the tangents at the node. We obtain as the only linear functions of $U, \tilde{H} U$ which are decomposable, $x^{3}+y^{3}$ and $x y z$, the equation $x y z=0$ gives $x y=0$ which belongs to the tangents at the node or else $z=0$, which is the equation of the line through the three inflexions: this line is so obtained a little further on in the text. 
It is interesting to verify this; I write $Z=-X-Y$, the cubic function then assumes the form

$$
\left(\alpha, \beta, \gamma, \delta \gamma(X, Y)^{3},\right.
$$

where $(\alpha, \beta, \gamma, \delta)$ have the values presently given.

The Hessian is

$$
\left(2 \alpha \gamma-2 \beta^{2}\right) X^{2}+(\alpha \delta-\beta \gamma) \cdot 2 X Y+\left(2 \beta \delta-2 \gamma^{2}\right) Y^{2},
$$

or writing $2 X Y=Z^{2}-X^{2}-Y^{2}$, this is

$$
=\left(2 \alpha \gamma-2 \beta^{2}-\alpha \delta+\beta \gamma\right) X^{2}+\left(2 \beta \delta-2 \gamma^{2}-\alpha \delta-\beta \gamma\right) Y^{2}+(\alpha \delta-\beta \gamma) Z^{2} .
$$

We find after some easy reductions,

$$
\begin{aligned}
& \frac{1}{3} \alpha=K^{\prime}+F^{\prime}, \quad=-3(a+c)(a c+M), \\
& \beta=-H^{\prime}+2 K^{\prime}+I^{\prime}+F^{\prime},=3 a(a+c)(b+c), \\
& \text { - } \gamma=-K^{\prime}+2 H^{\prime}+J^{\prime}+G^{\prime},=3 b(a+c)(b+c) \text {, } \\
& -\frac{1}{3} \delta=H^{\prime}+J^{\prime}, \quad=-3(b+c)(b c+M),
\end{aligned}
$$

and hence

$$
\alpha \delta-\beta \gamma=-81(a+c)(b+c)\{(a c+M)(b c+M)-a b(a+c)(b+c)\},
$$

where the expression in \{\} is

$$
\begin{aligned}
& =(a b+2 a c+b c)(a b+a c+2 b c)-a b\left(a b+a c+b c+c^{2}\right) \\
& =c\{a b(3 a+3 b)+c(b+2 a)(a+2 b)-a b(a+b+c)\} \\
& =2 c(a+b)(b c+c a+a b)
\end{aligned}
$$

and therefore

$$
\alpha \delta-\beta \gamma=-162(b+c)(c+a)(a+b) M c ;
$$

the other coefficients may be similarly calculated, and omitting the merely numerical factor, we have

$$
\text { Hessian }=(b+c)(c+a)(a+b) M\left(a X^{2}+b Y^{2}+c Z^{2}\right),
$$

which is right.

I write next

$$
\begin{aligned}
\tilde{H} U+3 M U-9 U= & X^{2}\left(-a 9 x+I^{\prime} Y-F^{\prime} Z\right) \\
& +Y^{2}\left(-b 9 y+J^{\prime} Z-G^{\prime} X\right) \\
& +Z^{2}\left(-a 9 z+K^{\prime} X-H^{\prime} Y\right)
\end{aligned}
$$


or writing $x=z-Y, y=z+X$, this is

$$
\begin{aligned}
\tilde{H} U+3 M U-9 U= & -9 z\left(a X^{2}+b Y^{2}+c Z^{2}\right) \\
& +X^{2}\left\{\left(I^{\prime}+a 9\right) Y-F^{\prime} Z\right\} \\
& +Y^{2}\left\{J^{\prime} Z-\left(G^{\prime}+b 9\right) X\right\} \\
& +Z^{2}\left\{K^{\prime} X-H^{\prime} Y\right\}
\end{aligned}
$$

we may determine 2 , so that the cubic function of $X, Y, Z$ contains the factor $a X^{2}+b Y^{2}+c Z^{2} ;$ writing $Z=-X-Y$, then

$$
\begin{aligned}
& \text { Contains the factor Quotient is } \\
& X^{3}\left(K^{\prime}+F^{\prime}\right)(a+c) X^{2}-3(a c+M) X \\
& +X^{2} Y\left(-H^{\prime}+2 K^{\prime}+F^{\prime}+I^{\prime}+a 9\right)+2 c X Y+3(b c+M) Y \text {. } \\
& +X Y^{2}\left(K^{\prime}-2 H^{\prime}-G^{\prime}-J^{\prime}-b 9\right)+(b+c) \quad Y^{2} \\
& +Y^{3} \quad\left(-J^{\prime}-H^{\prime}\right.
\end{aligned}
$$

We have seen that

$$
\begin{aligned}
& K^{\prime}+F^{\prime}=-3(a+c)(a c+M) \\
& J^{\prime}+H^{\prime}=-3(b+c)(b c+M)
\end{aligned}
$$

whence the quotient is, as above stated,

$$
=-3(a c+M) X+3(b c+M) Y \text {. }
$$

Comparing the coefficients of $X^{2} Y$, we have

$$
\begin{aligned}
a 9 & =-\left(-H^{\prime}+2 K^{\prime}+F^{\prime}+I^{\prime}\right)+3(a+c)(b c+M)-6 c(a c+M), \\
& =9 a(a+c)(b+c)+3(a+c)(a b+a c+2 b c)-6 c(a b+2 a c+b c), \\
& =12 a(b c+c a+a b)=12 a M,
\end{aligned}
$$

that is $9=12 M$; and the same value would have been obtained by comparing the coefficients of $X Y^{2}$. Hence $\tilde{H} U-9 M U$ divides by $a X^{2}+b Y^{2}+c Z^{2}$, the quotient being

which is

$$
-12 M z-3(a c+M) X+3(b c+M) Y,
$$

or, finally it is

$$
\begin{aligned}
& =-12 M z-3(a c+M)(y-z)+3(b c+M)(z-x), \\
& =-3\{(b c+M) x+(c a+M) y+(a b+M) z\},
\end{aligned}
$$

and we thus have

$$
\tilde{H} U-9 M U=-3\left(a X^{2}+b Y^{2}+c Z^{2}\right) \times\{(b c+M) x+(c a+M) y+(a b+M) z\},
$$

so that the three inflexions are the intersections of the cubic curve by the line

$$
(b c+M) x+(c a+M) y+(a b+M) z=0 .
$$

It may be noticed, that if we write

$$
\begin{aligned}
x+y+z & =u, \\
b c x+c a y+a b z & =-M u, \\
a x+b y+c z & =\quad v,
\end{aligned}
$$


then $x, y, z$ will be as

$$
\begin{aligned}
& (b-c)\{(2 M-b c) u-a v\} \\
: & (c-a)\{(2 M-c a) u-b v\} \\
: & (a-b)\{(2 M-a b) u-c v\},
\end{aligned}
$$

and substituting these values in the equation

$$
a x(y-z)^{2}+b y(z-x)^{2}+c z(x-y)^{2}=0
$$

of the cubic, we have a cubic equation for the ratio $(u: v)$; and thence the values $(x, y, z)$ for the coordinates of the inflexions.

It may be added, that we have

$$
\begin{aligned}
12 M U=-3\left(a X^{2}+b Y^{2}+c Z^{2}\right)\{(b c+M) x+(c a+M) y+(a b+M) z\} \\
+\left\{X^{2}\left(I^{\prime} Y-F^{\prime} Z\right)+Y^{2}\left(J^{\prime} Z-G^{\prime} X\right)+Z^{2}\left(K^{\prime} X-H^{\prime} Y\right)=0,\right.
\end{aligned}
$$

which is the equation of the cubic expressed in the canonical form.

Pp. 175-179. Effecting the process indicated p. 73, but writing for greater convenience $(x, y, z)$ in place of $(X, Y, Z)$, so that the substitution to be made is

$$
\begin{aligned}
& (a, b, c, f, \quad g, h, \quad i, j, k, \quad l \quad) \\
& =(6 x, 6 y, 6 z,-2 y,-2 z,-2 x,-2 z,-2 x,-2 y, x+y+z) \text {, }
\end{aligned}
$$

respectively (where I have corrected a misprint in the formula as originally given) I find the equation of the envelope to be

$$
\begin{aligned}
& 4 y z(y-z)^{2} \mathrm{a}^{4} \\
&+ 4 z x(z-x)^{2} \mathrm{~b}^{4} \\
&+ 4 x y(x-y)^{2} \mathrm{c}^{4} \\
&+ 4 z x\left(z^{2}+x^{2}+3 y z-2 z x+5 x y\right) \mathrm{b}^{3} \mathrm{c} \\
&+ 4 x y\left(x^{2}+y^{2}+3 z x-2 x y+5 y z\right) \mathrm{c}^{3} \mathrm{a} \\
&+ 4 y z\left(y^{2}+z^{2}+3 x y-2 y z+5 z x\right) \mathrm{a}^{3} \mathrm{~b} \\
&+ 4 x y\left(x^{2}+y^{2}+3 y z-2 x y+5 z x\right) \mathrm{bc}^{3} \\
&+ 4 y z\left(y^{2}+z^{2}+3 z x-2 y z+5 x y\right) \mathrm{ca}^{3} \\
&+ 4 z x\left(z^{2}+x^{2}+3 x y-2 z x+5 y z\right) \mathrm{ab}^{3} \\
&+ x\left(x^{3}-2 x^{2} y-2 x^{2} z+x y^{2}+38 x y z+x z^{2}+12 y^{2} z+12 y z^{2}\right) \mathrm{b}^{2} \mathrm{c}^{2} \\
&+ y\left(y^{3}-2 y^{2} z-2 y^{2} x+y z^{2}+38 x y z+y x^{2}+12 z^{2} x+12 z x^{2}\right) \mathrm{c}^{2} \mathrm{a}^{2} \\
&+ z\left(z^{3}-2 z^{2} x-2 z^{2} y+z x^{2}+38 x y z+z y^{2}+12 x^{2} y+12 x y^{2}\right) \mathrm{a}^{2} \mathrm{~b}^{2} \\
&+ 2 y z\left(11 x^{2}+y^{2}+z^{2}-2 y z+24 x y+24 z x\right) \mathrm{a}^{2} \mathrm{~b} c \\
&+ 2 z x\left(11 y^{2}+z^{2}+x^{2}-2 z x+24 y z+24 x y\right) \mathrm{b}^{2} \mathrm{ca} \\
&+ 2 x y\left(11 z^{2}+x^{2}+y^{2}-2 x y+24 z x+24 y z\right) \mathrm{c}^{2} \mathrm{a} b=0 . \\
&
\end{aligned}
$$

C. VI. 
The function on the left-hand side is the quotient by $-48(a+b+c)^{2}$ of the sextic function, Table 67 of my third Memoir on Quantics, [144]; the foregoing quotient was calculated without using the coefficient of the term in $a^{2} b^{2} c^{2}\left(\xi \eta^{2} \zeta^{2}\right)$ of the table, but by way of verification, I calculated from the table the term in question, and found it to be

$$
\begin{aligned}
& (x+y+z)^{4} \\
-\quad & 2(x+y+z)^{2}(y z+z x+x y) \\
+ & 296(x+y+z) x y z \\
-\quad & 8\left(y^{2} z^{2}+z^{2} x^{2}+x^{2} y^{2}\right),
\end{aligned}
$$

and this should consequently be equal to the coefficient of $a^{2} b^{2} c^{2}$ in the product of $(a+b+c)^{2}$ into the foregoing quartic function of $(a, b, c)$ that is, it should be

$$
\begin{aligned}
= & x\left(x^{3}-2 x^{2} y-2 x^{2} z+x y^{2}+38 x y z+x z^{2}+12 y^{2} z+12 y z^{2}\right) \\
& +y\left(y^{3}-2 y^{2} z-2 y^{2} x+y z^{2}+38 x y z+y x^{2}+12 z^{2} x+12 z x^{2}\right) \\
& +z\left(z^{3}-2 z^{2} x-2 z^{2} y+z x^{2}+38 x y z+z y^{3}+12 x^{2} y+12 x y^{2}\right) \\
& +4 y z\left(11 x^{2}+y^{2}+z^{2}-2 y z+24 x y+24 z x\right) \\
& +4 z x\left(11 y^{2}+z^{2}+x^{2}-2 z x+24 y z+24 x y\right) \\
& +4 x y\left(11 z^{2}+x^{2}+y^{2}-2 x y+24 z x+24 y z\right),
\end{aligned}
$$

which is accordingly found to be the case. 